\title{
Prevalência da Deficiência, Insuficiência de Vitamina D e Hiperparatiroidismo Secundário em Idosos Institucionalizados e Moradores na Comunidade da Cidade de São Paulo, Brasil
}

\section{RESUMO}

A ocorrência de fraturas osteoporóticas em idosos está relacionada às concentrações reduzidas de vitamina $D$ e conseqüente hiperparatiroidismo secundário, sendo os institucionalizados de maior risco. No Brasil, por seu alto grau de insolação, infere-se que a quantidade de vitamina $D$ da população seja adequada. Neste estudo, objetivamos avaliar as concentrações plasmáticas de 25-hidroxivitamina D (25OHD), paratormônio (PTH) e cálcio ionizado (Cai), assim como analisar a prevalência de hipovitaminose $D$ e de hiperparatiroidismo secundário em idosos moradores da cidade de São Paulo. Estudamos 177 pacientes institucionalizados (125 mulheres e 52 homens) com idade média (DP) de 76,6 $(9,0)$ anos, e 243 idosos ambulatoriais (168 mulheres e 75 homens) com 79,1 $(5,9)$ anos. Nesta avaliação, $71,2 \%$ do grupo institucionalizado e $43,8 \%$ do ambulatorial possuíam valores de $250 \mathrm{HD}$ menores do que o mínimo recomendado $(50 \mathrm{nmol} / \mathrm{l})$, sendo que as mulheres apresentaram valores consideravelmente mais baixos que os homens. 0 hiperparatiroidismo secundário ocorreu em $61,7 \%$ dos pacientes institucionalizados e em $54 \%$ dos ambulatoriais. Considerando os resultados obtidos, recomendamos a suplementação com doses eficientes de vitamina D para a população idosa brasileira, alem de sugerir uma discussão para a implementação de políticas de fortificação alimentar com vitamina $D$, especialmente direcionada àqueles com maior risco. (Arq Bras Endocrinol Metab 2007;51/3:437-442)

Descritores: Vitamina D; Hiperparatiroidismo secundário; Idosos; Deficiência; Insuficiência; 25-hidroxivitamina D; Epidemiologia

\section{ABSTRACT}

Prevalence of Vitamin D Deficiency, Insufficiency and Secondary Hyperparathyroidism in the Elderly Inpatients and Living in the Community of the City of São Paulo, Brazil.

The occurrence of osteoporotic fractures in the elderly is associated with reduced levels of vitamin $D$ and resulting secondary hyperparathyroidism, and inpatients are the ones at a higher risk. In Brazil, given its high level of insolation, the population's large amount of vitamin D is inferred to be adequate. In this study we aimed to assess the serum levels of 25-hydroxivitamin D (25OHD), parathormone (PTH) and ionized calcium (Cai), as well as to analyze the prevalence of both hypovitaminosis $\mathrm{D}$ and secondary hyperparathyroidism in the elderly living in the city of São Paulo. We studied 177 inpatients (125 women and 52 men) with mean age (SD) 76.6 (9.0) years, and 243 outpatients (168 women and 75 men) aged 79.1 (5.9) years. In this assessment $71.2 \%$ in the inpatients group and $43.8 \%$ in the outpatients group had $25 \mathrm{OHD}$ levels below the minimum recommended $(50 \mathrm{nmol} / \mathrm{l})$, with the women presenting with levels considerably lower than the men. Secondary hyperparathyroidism occurred in $61.7 \%$ of the inpatients and in $54 \%$ of the outpatients. Considering the results achieved, we recommend vitamin D supplementation in effective doses for the Brazilian elderly population, in addition to suggesting a discussion for the implementation of vitamin D-enhanced food policies, particularly oriented to the ones at a greater risk. (Arq Bras Endocrinol Metab 2007;51/3:437-442)

Keywords: Vitamin D; Secondary hyperparathyroidism; Elderly; Deficiency; Insufficiency; 25-hydroxivitamin D; Epidemiology artigo original

\author{
Gabriela LUPorinI SaRaiva \\ Maysa Seabra Cendoroglo \\ LUIZ ROBERTO RAMOS \\ Lara Miguel Quirino araújo \\ José Gilberto H. Vieira \\ Sérgio Setsuo Maeda \\ VICTÓRIA Z.C. BORBA \\ ILDA KUNII \\ LILLIAN F. HAYASHI \\ MARISE LAZARETtI-CASTRO
}

Departamento de

Endocrinologia (GLS, JGHV, SSM, IK, LFH \& ML-C) e Departamento de Geriatria (MSC, LRR \& LMQA), Escola Paulista de Medicina, Universidade Federal de São Paulo, SP, e Serviço de Endocrinologia da Universidade Federal do Paraná (VZCB), Curitiba, PR.

Recebido em 11/05/05

Revisado em 07/11/06

Aceito em 17/11/06 


\section{Deficiência de Vitamina D em Idosos Saraiva et al.}

A VITAMINA D3, ou COLECALCIFEROL, é um hormônio sintetizado na pele através de uma reação de isomerização catalisada pela radiação ultravioleta (UV) $(290$ a $315 \mathrm{~nm}$ ) (1). A sua forma ativa [calcitriol ou 1,25(OH)2D3] estimula a absorção intestinal de cálcio, atua sobre a mineralização óssea (2) e regula a síntese e secreção do paratormônio (PTH) (3). Uma queda na concentração sérica de vitamina D leva a uma absorção insuficiente de cálcio que se reflete no cálcio ionizado (Cai) circulante. A alteração do cálcio circulante é imediatamente percebida pelos sensores de cálcio nas paratiróides que, com o objetivo de manter a calcemia dentro da faixa de normalidade, aumentam a síntese de PTH (4). Esse hiperparatiroidismo secundário à hipovitaminose $\mathrm{D}$ é responsável por um comprometimento da qualidade óssea, associando-se a um maior risco de fraturas $(2,5)$.

A população geriátrica é mais sensível à hipovitaminose $\mathrm{D}$ por vários motivos, dentre eles por se expor menos ao sol, ter sua capacidade de produção cutânea de vitamina D reduzida (6-8), alimentar-se de forma inadequada (9-12), absorver menos vitamina D pelo trato gastrintestinal (3), usar múltiplas drogas que interferem na absorção/metabolização da vitamina D e apresentar comprometimento renal (3). Dentro deste grupo, pacientes institucionalizados têm um risco ainda maior para esta situação (13), que se reflete em um aumento de até 10,5 vezes no risco de fratura (14).

E embora toda literatura seja unânime na constatação da elevada prevalência de deficiência deste hormônio no idoso com conseqüente aparecimento do hiperparatiroidismo secundário e aumento do risco de fraturas, não existem estudos populacionais consistentes em nosso meio que tenham avaliado o estado nutricional de vitamina D. Neste estudo, verificamos a prevalência da hipovitaminose D e do hiperparatiroidismo secundário em uma amostra de pacientes geriátricos institucionalizados e moradores da comunidade de uma região subtropical $\left(23,3^{\circ} \mathrm{S}\right)$, onde a incidência solar é considerada abundante, mesmo nos meses de inverno.

\section{CASUÍSTICA}

Foram selecionados pacientes institucionalizados há pelo menos 6 meses e ambulatoriais com mais de 65 anos, que previamente não apresentassem alteração renal ou hepática significativa, não fossem etilistas ativos, deambulassem e não tivessem história de imobilização nos últimos seis meses.

De um total de 676 pacientes internados em 2 instituições geriátricas da cidade de São Paulo há pelo menos 6 meses, 479 não preenchiam os critérios de inclusão ou recusaram-se a participar do trabalho. $\mathrm{O}$ principal motivo de exclusão foi não deambularem.

O grupo ambulatorial foi constituído a partir de um estudo de coorte (EPIDOSO) entre moradores no subdistrito da Saúde, bairro que engloba a Universidade Federal de São Paulo, e corresponde a um dos 55 subdistritos da cidade de São Paulo. Essa região da cidade caracteriza-se por comportar uma população em sua maioria caucasiana, de diferentes níveis sócioeconômicos (15). No inicio do estudo (1991), foi realizado um censo e todos os indivíduos moradores neste subdistrito, que tivessem 65 anos ou mais, foram cadastrados perfazendo um total de 1667 idosos. A cada 2 anos esses indivíduos eram contatados e reavaliados. Dez anos depois, 288 indivíduos da coorte inicial compareceram ao ambulatório (2) e, dentre estes, 250 tiveram os seus dados colhidos para o presente estudo.

Cento e noventa e sete indivíduos institucionalizados e 250 pacientes ambulatoriais tiveram seus dados clínicos obtidos e amostra de sangue coletada. Desses, 17 pacientes foram excluídos das análises finais por apresentarem hipercalcemia $(n=12)$, creatinina $>2$ $\mathrm{mg} / \mathrm{dL}(\mathrm{n}=4)$ e hipocalcemia $(\mathrm{n}=1)$ na ocasião da análise das amostras de sangue. Restaram para as análises finais 187 indivíduos do grupo institucionalizado e 243 do grupo ambulatorial.

Para complementar nossas informações sobre os valores de normalidade para os métodos laboratoriais, foi criado um terceiro grupo de 141 voluntários jovens e saudáveis composto de 97 mulheres e 44 homens com idade média de 26,4 $(5,0)$ anos, utilizado como grupo controle.

\section{METODOLOGIA}

O protocolo de estudo e o termo de consentimento informado foi previamente aprovado pelo comitê de ética do Hospital São Paulo/ Universidade Federal de São Paulo. Cada participante ou seu responsável forneceu consentimento formal para sua participação no estudo.

Amostras de sangue em jejum foram colhidas para mensuração de cálcio ionizado, PTH, 25-hidroxivitamina D (25OHD), albumina e creatinina. Dos participantes, foi obtido histórico médico e medidas antropométricas. A coleta de sangue dos pacientes do grupo institucionalizado ocorreu entre o fim do outono e o início do inverno, enquanto que as coletas do grupo ambulatorial e controle foram realizadas ao longo do ano.

A dosagem de cálcio ionizado foi realizada logo após a coleta e o restante da amostra foi centrifugado a $1.200 \mathrm{rpm}$ por 10 minutos a uma temperatura de $4^{\circ} \mathrm{C}$. O soro foi armazenado a $-70^{\circ} \mathrm{C}$ até o momento da análise. 


\section{Avaliação laboratorial}

A albumina e a creatinina foram avaliadas através de equipamento colorimétrico automático (Modular Roche, São Paulo, Brasil). As dosagens de 25OHD foram feitas utilizandose um kit de radioimunoensaio comercial (Nichols Institute Diagnostics, CA, USA) que reconhece tanto a $25 \mathrm{OHD}_{2}$ quanto a $25 \mathrm{OHD}_{3}$ (coeficiente de variação inter-ensaio de $9,7 \%$; coeficiente de variação intra-ensaio de $16,5 \%$ ).

Os pacientes foram classificados como deficientes, insuficientes ou suficientes em vitamina $\mathrm{D}$ de acordo com a classificação de McKenna e Freaney (16). Segundo estes autores, deficientes em vitamina $\mathrm{D}$ são os pacientes com valores de $25 \mathrm{OHD}<25 \mathrm{nmol} / \mathrm{l}$ e insuficientes, aqueles com valores entre 25 e $50 \mathrm{nmol} / \mathrm{l}$. Aqueles com concentrações plasmáticas $>100$ nmol/1 são considerados como plenamente suficientes. No presente artigo, o termo hipovitaminose $\mathrm{D}$ refere-se ao conjunto de pacientes com $25 \mathrm{OHD} \leq 50 \mathrm{nmol} / \mathrm{l}$.

O PTH intacto foi analisado através de um ensaio imunofluorimétrico desenvolvido em nosso laboratório (17). Foram considerados portadores de hiperparatiroidismo secundário aqueles indivíduos que possuíam PTH acima da normalidade na presença de valores de cálcio ionizado normais. As dosagens de Cai foram feitas pelo método eletrodo-específico em equipamento automático (AVL 984$S, M N$, USA).

\section{Hábitos de vida e história médica}

Os grupos foram avaliados utilizando-se um questionáriopadrão aplicado por um médico previamente treinado. $\mathrm{O}$ paciente era questionado sobre tabagismo, etilismo, uso corrente de diuréticos tiazídicos, anticonvulsivantes, antiinflamatórios não-hormonais, corticosteróides, bisfosfonatos e suplementos de cálcio ou vitamina $\mathrm{D}$ e a presença de comorbidades segundo o relato do próprio paciente ou descrição de prontuário.

\section{Análise estatística}

Os resultados estão expressos em média (desvio-padrão) ou porcentagens. Os 2 grupos de idosos foram comparados entre si utilizando-se o teste $t$ de Student, análise de variância (ANOVA) ou teste de Chi-quadrado, quando aplicável. As correlações foram estabelecidas através do coeficiente linear de Pearson. Considerou-se um p significante se $<0,05$. Para avaliação das variáveis interferentes nas concentrações de PTH e 25OHD foram feitas análises de múltiplas variáveis.

\section{RESULTADOS}

A análise dos dados foi realizada com 177 pacientes do grupo institucionalizado ( 125 mulheres e 52 homens) com idade média de $76,6(9,0)$ anos e 243 pacientes (168 mulheres e 75 homens) com idade média de 79,1 $(5,9)$ anos pertencentes ao grupo ambulatorial. Não houve diferença significativa entre os grupos quanto à distribuição por sexo, idade, índice de massa corpórea ou presença de co-morbidades. O grupo institucionalizado apresentava maior prevalência de negros $(9,0 \% \mathrm{e}$ $4,2 \%$ respectivamente) e fumantes $(34 \%$ e $5,9 \%$ respectivamente), utilizavam mais anticonvulsivantes ( $7,9 \%$ e $2,5 \%$ respectivamente), antiinflamatórios nãohormonais $(29,4 \%$ e $7,4 \%$ respectivamente) e corticosteróides $(4,0 \%$ e 1,6\% respectivamente) do que o grupo ambulatorial. $\mathrm{O}$ grupo ambulatorial, por sua vez, utilizava diuréticos tiazídicos $(23,0 \%$ e $15,8 \%$ respectivamente), bisfosfonatos $(3,7 \%$ e $1,1 \%$ respectivamente), cálcio oral ( $15,6 \%$ e $3,4 \%$ respectivamente) e vitamina $\mathrm{D}$ oral $(9,5 \%$ e $4,0 \%$ respectivamente $)$ com maior freqüência do que o grupo institucionalizado.

Os resultados da avaliação laboratorial estão descritos na tabela 1 . O grupo ambulatorial apresentou, em média, maior concentração sérica de $25 \mathrm{OHD}$ em relação ao grupo institucionalizado. Esta diferença se manteve quando apenas os indivíduos que colheram sangue na mesma época do ano (outono) foram comparados entre si $(\mathrm{p}<0,001)$ ou quando homens e mulheres eram analisados separadamente $(\mathrm{p}=0,006$ e $\mathrm{p}<$ 0,001 , respectivamente). O uso de suplementos de vitamina $\mathrm{D}$ em doses que variaram de $125 \mathrm{UI} /$ dia a

Tabela 1. Resultados das análises laboratoriais dos grupos controle e de idosos institucionalizados e ambulatoriais, expressos em média (DP).

\begin{tabular}{|c|c|c|c|c|c|}
\hline & Institucionalizados & Ambulatoriais & $\mathbf{p}^{1}$ & Controle & $\begin{array}{l}\text { Valores de } \\
\text { referência }\end{array}$ \\
\hline Glicemia & $93,5(25,6)$ & $99,6(25,8)$ & 0,021 & $77,54(15,2)$ & $70-100 \mathrm{mg} / \mathrm{dl}$ \\
\hline Creatinina & $1,0(0,2)$ & $1,0(0,3)$ & $<0,001$ & $1,0(0,3)$ & $\begin{array}{c}1,0 \mathrm{mg} / \mathrm{dl} \text { (mulheres) } \\
1,2 \mathrm{mg} / \mathrm{dl} \text { (homens) }\end{array}$ \\
\hline Albumina & $3,7(0,4)$ & $4,1(0,3)$ & $<0,001$ & $4,4(0,7)$ & $3,5-5,0 \mathrm{~g} / \mathrm{dl}$ \\
\hline Ca i & $1,29(0,04)$ & $1,26(0,05)$ & $<0,001$ & $1,27(0,08)$ & $1,20-1,40 \mathrm{mM}$ \\
\hline 25OHD & $36,5(23,5)$ & $49,5(28,5)$ & $<0,001$ & $81,9(34,4)$ & \\
\hline PTH & $96,8(58,5)$ & $82,5(43,5)$ & 0,009 & $36,4(22,9)$ & $10-70 \mathrm{pg} / \mathrm{ml}$ \\
\hline
\end{tabular}

1 Valores de p são referentes à comparação entre o grupo institucionalizado e o ambulatorial. 
$1.000 \mathrm{UI} /$ dia ocorreu em 6,7\% da amostra, e a $25 \mathrm{OHD}$ destes pacientes não foi diferente da dos que não utilizavam suplementos $(\mathrm{p}=0,172)$. Entretanto, ambos os grupos apresentaram valores médios de 25OHD muito inferiores aos observados na população jovem utilizada como controle.

Dentre os pacientes institucionalizados, 40,7\% eram portadores de deficiência e $30,5 \%$ deles de insuficiência de vitamina D. No grupo ambulatorial, $15,8 \%$ dos pacientes eram portadores de deficiência e $40,0 \%$, de insuficiência de vitamina D. Apenas 1,2\% dos pacientes do institucionalizado e $4,2 \%$ dos do ambulatorial apresentavam valores considerados ideais (> $100 \mathrm{nmol} / \mathrm{l}$ ) de 25OHD.

Em ambos os grupos, as mulheres tinham valores de $25 \mathrm{OHD}$ consideravelmente mais baixos do que os homens $(\mathrm{p}<0,001)$. Como era de se esperar, observou-se uma correlação negativa entre $25 \mathrm{OHD}$ e o PTH $(r=-0,41)$. Para excluir uma possível interferência da função renal reduzida sobre as concentrações de PTH, excluímos desta análise todos aqueles com creatinina sérica alterada. Considerando-se apenas os idosos com valores de creatinina sérica normais ( $\leq 1,0 \mathrm{mg} / \mathrm{dL}$ para mulheres $\mathrm{e} \leq 1,2 \mathrm{mg} / \mathrm{dL}$ para homens), observamos que $88,9 \%$ dos pacientes com a $25 \mathrm{OHD}$ acima de $100 \mathrm{nmol} / 1$ possuíam o $\mathrm{PTH}$ dentro dos valores de normalidade, enquanto que $63,9 \%$ dos pacientes com 25OHD menor que 25 nmol/l apresentavam PTH elevado (figura 1 ).

$\mathrm{O}$ hiperparatiroidismo secundário ocorreu em $61,7 \%$ dos pacientes do grupo institucionalizado e em $54 \%$ dos pacientes do grupo ambulatorial. Na análise de múltiplas variáveis, os valores de PTH mostraram

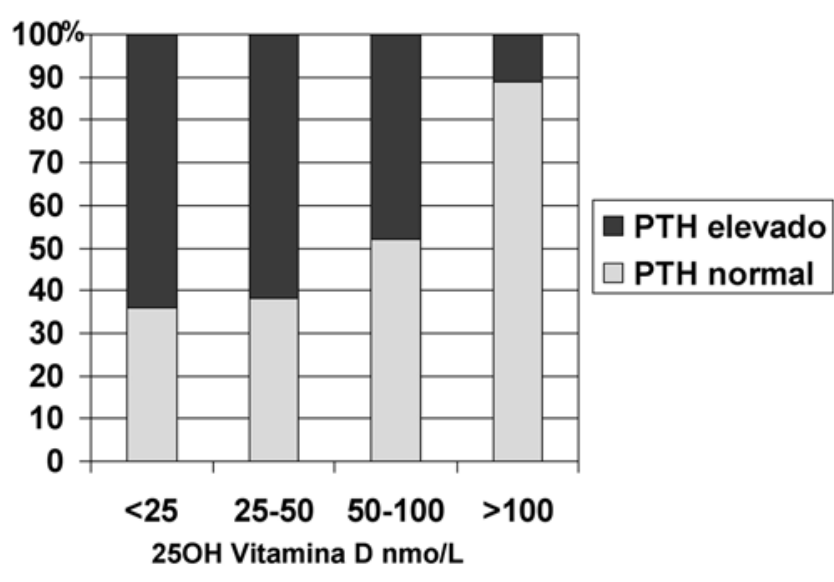

Figura 1. Distribuição percentual dos pacientes com valores normais e elevados de PTH segundo as concentrações de 250HD. Nesta análise foram incluídos apenas indivíduos com valores de creatinina plasmática dentro da normalidade sofrer influência da origem do paciente (ambulatorial $\mathrm{x}$ institucionalizado), da creatinina sérica, do cálcio ionizado e da concentração sérica de 25OHD. Apenas a origem e sexo do indivíduo influenciaram a concentração sérica de $25 \mathrm{OHD}$. Não houve correlação desta com os outros parâmetros avaliados (idade, IMC, tempo de internação, creatinina, albumina, cálcio ionizado ou PTH).

\section{DISCUSSĀO}

A deficiência de vitamina $\mathrm{D}$ está associada a uma menor mineralização do tecido osteóide caracterizando a osteomalácia $(8,11)$. Entretanto, análises mais recentes demonstram que valores não tão reduzidos de vitamina $\mathrm{D}$ estão associados a aumento do turnover ósseo e conseqüente aumento de fraturas. Portanto, valores intermediários anteriormente considerados "normais", embora suficientes para prevenir a osteomalácia, foram progressivamente sendo responsabilizados por fenômenos fisiopatológicos como o hiperparatiroidismo secundário e risco de fratura aumentado (18). Atualmente, existe uma grande discussão na literatura sobre quais valores de $25 \mathrm{OHD}$ devam ser considerados normais, uma vez que múltiplas variáveis podem interferir nessas concentrações, como idade, localização geográfica, sexo, atividade ocupacional, entre outras. Adotamos a classificação de McKenna e Freaney por ser a de maior consenso na literatura de hoje.

Dados mundiais mostram que $5 \%$ a $25 \%$ da população idosa independente e 60 a $80 \%$ dos pacientes institucionalizados (19) são deficientes ou insuficientes em vitamina D. Considerando que a maior fonte de vitamina $\mathrm{D}$ é oriunda da exposição ao sol, a alta prevalência de hipovitaminose D encontrada tanto em pacientes ambulatoriais quanto em institucionalizados de nossa região, considerada de alta incidência solar, foi surpreendente. Essa prevalência foi maior que a de países de latitude mais alta como a Nova Zelândia (20), Inglaterra ou Alemanha (19), sendo as mulheres mais afetadas que os homens $(77,6 \%$ e $45,9 \%$ respectivamente). Essa inversão inesperada entre latitude e prevalência de hipovitaminose D também foi encontrada no estudo MORE (Multiple Outcomes Raloxifene Evaluation), fato atribuído não à latitude, mas às políticas de enriquecimento alimentar.

Os valores de PTH correlacionam-se de maneira inversa às concentrações de vitamina $\mathrm{D}$ tanto em idosos quanto na população jovem, e a alta prevalência 
de hipovitaminose D se correlacionou com a presença de hiperparatiroidismo secundário. Segundo nossos resultados, assim como os de outros autores $(21,22)$, raramente vimos valores de PTH elevados quando as concentrações séricas de $25 \mathrm{OHD}$ atingiam $100 \mathrm{nmol} / \mathrm{l}$. Por outro lado, no grupo controle constituído por jovens saudáveis, os valores médios de $25 \mathrm{OHD}$ de $82 \mathrm{nmol} / 1$ foram suficientes para manter o PTH dentro da faixa de normalidade. Essa constatação confirma os conceitos atuais de que os idosos necessitam de concentrações mais elevadas de 25OHD para normalizarem suas concentrações plasmáticas de PTH (23).

$\mathrm{Na}$ tentativa de se identificar quais os fatores de risco nessa população para a hipovitaminose $\mathrm{D}$ e para o hiperparatiroidismo secundário, foi realizada a análise de múltiplas variáveis onde o fato de ser ou não institucionalizado foi o que mais influenciou nesses resultados, seguido pelo sexo, sendo que o feminino apresenta com maior tendência a valores baixos. A população institucionalizada possuía mais pacientes negros e usuários de anticonvulsivantes, características que podem estar prejudicando a concentração plasmática de vitamina D. Por outro lado, tiveram a coleta realizada no outono, estação do ano onde a concentração de vitamina $\mathrm{D}$ atinge seu maior valor, conseqüente à exposição ao sol intensificada no verão (24). A creatinina, o cálcio ionizado e a $250 \mathrm{OD}$ foram as variáveis que mais influenciaram o PTH.

Sabendo-se que a reposição de cálcio e vitamina D tem efeito protetor sobre a massa óssea (25-29), torna-se relevante a identificação de pacientes com risco de hipovitaminose $\mathrm{D}$, para que haja uma adequada reposição atuando como mecanismo preventivo sobre o risco de fratura.

O RDA (Recommended Dietary Allowance) recomenda uma ingestão diária de 400 UI para pacientes entre 50 e 70 anos e de 600 UI para aqueles com mais de 70 anos, o que corresponde a 20 minutos de exposição diária de $5 \%$ da superfície corpórea. As fontes alimentares de vitamina D são pobres, e os alimentos mais ricos neste pré-hormônio (peixes de águas frias e cogumelos) não fazem parte de nosso cardápio habitual.

A reposição de 400 UI ao dia de $250 H D$ corresponde a uma $25 \mathrm{OHD}$ sérica de $38 \mathrm{nmol} / \mathrm{l}$ (7), ou seja, uma concentração aquém do desejado, e não demonstrou efeito protetor em idosos quanto à incidência de fraturas (30). Reposições de 700-800 UI por dia foram responsáveis por uma redução de até $43 \%$ na incidência de fraturas de colo de fêmur (3133). O uso de 1000 UI diárias também não foi capaz de trazer os valores de $25 \mathrm{OHD}$ para a normalidade, em idosos institucionalizados (34). Quando se detecta concentrações deficientes ou insuficientes de 25OHD no plasma, a correção inicial deve ser feita com doses mais elevadas de vitamina D, como 50.000 UI de colecalciferol semanal, ao longo de 6 semanas, para então passar para a dose de manutenção, que deve ficar por volta de 7.000 a 14.000 UI semanais. No Brasil, a maioria dos polivitamínicos direcionados à população geriátrica, além de cara, contém apenas 200 a 400 UI de vitamina D. Portanto, no caso de idosos, a única possibilidade de se utilizar colecalciferol isolado nas doses necessárias é o uso de formulações magistrais. A política de fortificação de alimentos como leite, suco de laranja e manteiga, com vitamina $\mathrm{D}$ demonstrou ser eficiente para elevar as concentrações plasmáticas de 25OHD nos países em que é utilizada. Políticas semelhantes poderiam ser estudadas também em nosso meio, com benefícios potenciais para nossa população geriátrica como demonstrado em nosso trabalho.

Chamamos atenção, ainda, da nossa comunidade médica para o risco de uma interpretação equivocada para os valores elevados de PTH em idosos, que podem erroneamente ser diagnosticado como hiperparatiroidismo primário. Nessa situação, a presença de concentrações plasmáticas normais ou reduzidas de cálcio deve remeter à investigação do status nutricional de vitamina $\mathrm{D}$, para sua possível correção e posterior normalização dos valores de PTH.

Concluindo, observamos elevada prevalência de deficiência e insuficiência de vitamina D em nossa população geriátrica, que foi mais acentuada no grupo institucionalizado, mas também bastante evidente dentre os moradores da comunidade. Em função disso, acreditamos que o incentivo à exposição solar, à suplementação medicamentosa com colecalciferol e o enriquecimento alimentar sejam condutas de saúde pública que devem ser incentivadas em nosso país.

\section{AGRADECIMENTOS}

À FAPESP (Fundo de Apoio à Pesquisa do Estado de São Paulo) (01/09027-1) pelo suporte financeiro, a Maria Cristina Carnevale pelas traduções ao inglês, e a Fábio Tadeu Montesano pelo suporte e análise estatística.

\section{REFERÊNCIAS}

1. Rapuri PB, Kinyamu HK, Gallagher JC, Haynatzka V. Seasonal changes in calciotropic hormones, bone markers and bone mineral density in elderly women. J Clin Endocrinol Metab 2002;87(5):2024-32. 


\section{Deficiência de Vitamina D em Idosos}

Saraiva et al.

2. Ramos LR, Toniolo J, Cendoroglo MS, Garcia JT, Najas MS, Perracini M, et al. Two-year follow-up study of elderly residents in S. Paulo, Brazil: methodology and preliminary results. Rev Saude Pública 1998;32(5):397-407.

3. Gloth III FM, Tobin JD. Vitamin D deficiency in older people. J Am Geriatr Soc 1995:43:822-8.

4. Goulding A. Lightening the fracture load: growing evidence suggests many older New Zealanders would benefit from more vitamin D. N Z Med J 1999:112(1095):329-30.

5. Bettica $P$, Bervilacgue $M$, Vago T. High prevalence of hypovitaminosis $D$ among free-living post-menopausal women refered to an osteoporosis out patient clinic in northern Italy for initial screening. Osteoporos Int 1999;9:226-9.

6. Vieth R. Vitamin D supplementation, 25-hydroxyvitamin D concentrations, and safety. Am J Clin Nutr 1999;69:842-56.

7. LeBoff MS, Kohlmeier L, Hurwitz S, Franklin J, Wright J, Glowacki J. Occult vitamin D deficiency in postmenopausal US women with acute hip fracture. JAMA 1999;281(16): 1505-11

8. Pfeifer M, Begerow B, Minne HW. Vitamin D and muscle function. Osteoporos Int 2002;13:187-94.

9. Bates CJ, Prentice A, Cole TJ, Pols JC, Doyle W, Finch S, et al. Micronutrients: highlights and research challenges from the 1994-1995 National Diet and Nutrition Survey of people aged 65 years and over. Br J Nutr 1999;82:7-15.

10. Utiger R. The need for more vitamin D - Editorials. N Engl J Med 1998;338(12):828-9.

11. Robins SP, New S. Symposium on Nutritional Aspects of Bone. Proc Nutr Soc 1997:56:903-14.

12. Kudlacek S, Schneider B, Peterlik M, Leb G, Klaushofer K, Weber K, et al. Assessment of vitamin D and calcium status in healthy adult Austrians. Eur J Clin Invest 2003:33(4):323-31.

13. O'Dowd KJ, Clemens TL, Kelsey JL, Lindsay R. Exogenous calciferol (vitamin $\mathrm{D}$ ) and vitamin $\mathrm{D}$ endocrine status among elderly nursing home residents in the New York City area. $\mathbf{J}$ Am Geriatr Soc 1993:41:414-21.

14. Rasmussen LB, Hansen GL, Hansen E, Koch B, Mosekilde L, Molgaard C, et al. Vitamin D: should the supply in the Danish population be increased? Int J Food Sci Nutr 2000;51:20915.

15. Ramos LR, Simões EJ, Albert MS. Dependence in activities of daily living and cognitive impairment strongly predicted mortality in older urban residents in Brazil: 2-year follow-up. J Am Geriatr Soc 2001;49:1168-75.

16. McKenna MJ, Freaney R. Secondary hyperparthyroidism in the elderly: means to defining hypovitaminosis D. Osteoporos Int 1998;8(suppl. 2):S3-6.

17. Vieira JG, Nishida SK, Kasamatsu TS, Amarante EC, Kunii IS. Development and clinical appication of na immunofluorometric assay for intact parathyroid hormone. Braz J Med Biol Res 1994;27(10):2379-82.

18. Lips P. Which circulating level of 25-hydroxyvitamin D is appropriate? J Steroid Biochem Mol Biol 2004;89(90):611-4.

19. Lips $P$, Duong $T$, Oleksik A, Black D, Cummings $S$, Cox $D$, et al. A global study of vitamin $D$ status and parathyroid function in postmenopausal women with osteoporosis: baseline data from the Multiple Outcomes of Raloxifen Evaluation clinical trial. J Clin Endocrinol Metab 2001:86(3):1212-21.

20. McAuley KA, Jones S, Lewis-Barned NJ, Manning P, Goulding $A$. Low vitamin $D$ status Is common among elderly Dunedin women. N Z Med J 1997;110:275-7.

21. Cannata-Andia JB, Alonso CG. Vitamin D deficiency: a neglected aspect of disturbed calcium metabolism in renal failure. Nephrol Dial Trasplant 2002;17(11):1875-8.

22. Patel R, Collins D, Bullock S, Swaminathan R, Blake GM, Fogelman I. The effect of season and vitamin D supplementation on bone mineral density in healthy women: a doublemasked crossover study. Osteoporos Int 2001;12:319-25.
23. Vieth $R$, Ladak $Y$, Walfish PG. Age-related changes in the 25hydroxyvitamin $\mathrm{D}$ versus parathyroid hormone relationship suggest a different reason why older adults require more vitamin D. J Clin Endocrinol Metab 2003;88(1):185-91.

24. Saraiva GL, Cendoroglo MS, Ramos LR, Araújo LMQ, Vieira $\mathrm{JGH}$, Kunii I, et al. Influence of ultraviolet radiation on the production of 25-hydroxivitamin $D$ in the elderly population in the city of São Paulo $\left(23^{\circ} 34^{\prime}\right.$ s), Brazil. Osteoporos Int 2005:16(12):1649-54.

25. Compston JE. The role of vitamin D and calcium suplementation in the prevention of osteoporotic fratures in the elderly. Clin Endocrinol 1995;43:393-405.

26. Krieg MA, Jacque AF, Brengartner M, Cuttelod S, Thiébaud D, Burckhardt P. Effect of supplementation with vitamin D3 and calcium on quantitative ultrasound of bone in elderly institutionalized women: a longitudinal study. Osteoporosis Int 1999;9:483-8.

27. Bauer DC, Palermo L, Black D, Cauley JA. Quantitative ultrasound and mortality: a prospective study. Osteoporos Int 2002:13:606-12.

28. Suleiman S, Nelson M, Li F, Buxton-Thomas M, Moniz C. Effect of calcium intake and physical activity level on bone mass and turnover in healthy, white, postmenopausal women. Am J Clin Nutr 1997;66:937-43.

29. Grados F, Brazier M, Kamel S, Mathieu M, Hurtebize N, Maamer M, et al. Prediction of bone mass density variation by bone remodeling markers in postmenopausal women with vitamin $D$ insufficiency treated with calcium and vitamin D supplementation. J Clin Endocrinol Metab 2003; 88(11):5175-9.

30. Lips P. Vitamin D deficiency and secondary hyperparathyroidism in the elderly: consequences for bone loss and fractures and therapeutic implications. Endocr Rev 2001;22(4):477-501.

31. Chapuy MC, Arlot ME, Delmas PD, Meunier PJ. Effect of calcium and cholecalciferol treatmente for three years on hip fractures in elderly women. BMJ 1994;308(6936):1081-2.

32. Dawson-Hughes B, Harris S, Krall EA, Dallal GE. Effect of calcium and vitamin $\mathrm{D}$ supplementation on bone density in men and women 65 years of age or older. N Engl J Med 1997;337(10):670-6.

33. Trived P, Doll R. Effect of four monthly oral vitamin D3 (cholecalciferol) supplementation on fractures and mortality in men and women living in the community: randomised double blind controlled trial. BMJ 2003;326(7387):469.

34. Canto-Costa MHS, Kunii I, Hauache OM. Avaliação dos níveis de 25-hidroxivitamina $D$ e de parâmetros de adiposidade como determinantes para adequação de suplementação de colecalciferol para idosos institucionalizados (máster tesis).

\section{Endereço para correspondência:}

Gabriela Luporini Saraiva

Departamento de Endocrinologia

Escola Paulista de Medicina,

Universidade Federal de São Paulo

Caixa Postal 20266

04034-970 São Paulo, SP

Fax: (11) 5579-6636

E-mail: gabrielasaraiva@uol.com.br 